\title{
Finite summation formulas involving binomial coefficients, harmonic numbers and generalized harmonic numbers
}

Junesang Choi*

\section{"Correspondence:}

junesang@mail.dongguk.ac.kr Department of Mathematics, Dongguk University, Gyeongju, 780-714, Republic of Korea

\begin{abstract}
A variety of identities involving harmonic numbers and generalized harmonic numbers have been investigated since the distant past and involved in a wide range of diverse fields such as analysis of algorithms in computer science, various branches of number theory, elementary particle physics and theoretical physics. Here we show how one can obtain further interesting identities about certain finite series involving binomial coefficients, harmonic numbers and generalized harmonic numbers by applying the usual differential operator to a known identity.
\end{abstract}

MSC: Primary 11M06; 33B15; 33E20; secondary 11M35; 11M41; 40C15

Keywords: harmonic numbers; generalized harmonic numbers; Riemann zeta function; Hurwitz zeta function; Stirling numbers of the first kind; generalized hypergeometric function ${ }_{p} F_{q}$; summation formulas for ${ }_{p} F_{q}$; psi-function; polygamma functions

\section{Introduction and preliminaries}

The generalized harmonic numbers $H_{n}^{(s)}$ of order $s$ are defined by (cf. [1]; see also [2, 3], [4, p.156] and [5, Section 3.5])

$$
H_{n}^{(s)}:=\sum_{j=1}^{n} \frac{1}{j^{s}} \quad(n \in \mathbb{N} ; s \in \mathbb{C}),
$$

and

$$
H_{n}:=H_{n}^{(1)}=\sum_{j=1}^{n} \frac{1}{j} \quad(n \in \mathbb{N})
$$

are the harmonic numbers. Here $\mathbb{N}$ and $\mathbb{C}$ denote the set of positive integers and the set of complex numbers, respectively, and we assume that

$$
H_{0}:=0, \quad H_{0}^{(s)}:=0 \quad(s \in \mathbb{C} \backslash\{0\}) \quad \text { and } \quad H_{0}^{(0)}:=1 .
$$

The generalized harmonic functions $H_{n}^{(s)}(z)$ are defined by (see [2, 6]; see also [7, 8])

$$
H_{n}^{(s)}(z):=\sum_{j=1}^{n} \frac{1}{(j+z)^{s}} \quad\left(n \in \mathbb{N} ; s \in \mathbb{C} \backslash \mathbb{Z}^{-} ; \mathbb{Z}^{-}:=\{-1,-2,-3, \ldots\}\right)
$$

\section{Springer}

(0) 2013 Choi; licensee Springer. This is an Open Access article distributed under the terms of the Creative Commons Attribution License (http://creativecommons.org/licenses/by/2.0), which permits unrestricted use, distribution, and reproduction in any medium, provided the original work is properly cited. 
so that, obviously,

$$
H_{n}^{(s)}(0)=H_{n}^{(s)}
$$

Equation (1.1) can be written in the following form:

$$
H_{n}^{(s)}=\zeta(s)-\zeta(s, n+1) \quad(\Re(s)>1 ; n \in \mathbb{N})
$$

by recalling the well-known (easily-derivable) relationship between the Riemann zeta function $\zeta(s)$ and the Hurwitz (or generalized) zeta function $\zeta(s, a)$ (see [4, Eq. 2.3(9)])

$$
\zeta(s)=\zeta(s, n+1)+\sum_{k=1}^{n} k^{-s} \quad\left(n \in \mathbb{N}_{0}:=\mathbb{N} \cup\{0\}\right)
$$

The polygamma functions $\psi^{(n)}(s)(n \in \mathbb{N})$ are defined by

$$
\psi^{(n)}(s):=\frac{d^{n+1}}{d z^{n+1}} \log \Gamma(s)=\frac{d^{n}}{d s^{n}} \psi(s) \quad\left(n \in \mathbb{N}_{0} ; s \in \mathbb{C} \backslash \mathbb{Z}_{0}^{-}:=\mathbb{Z}^{-} \cup\{0\}\right),
$$

where $\Gamma(s)$ is the familiar gamma function, and the psi-function $\psi$ is defined by

$$
\psi(s):=\frac{d}{d s} \log \Gamma(s) \quad \text { and } \quad \psi^{(0)}(s)=\psi(s) \quad\left(s \in \mathbb{C} \backslash \mathbb{Z}_{0}^{-}\right) .
$$

A well-known (and potentially useful) relationship between the polygamma functions $\psi^{(n)}(s)$ and the generalized zeta function $\zeta(s, a)$ is given by

$$
\psi^{(n)}(s)=(-1)^{n+1} n ! \sum_{k=0}^{\infty} \frac{1}{(k+s)^{n+1}}=(-1)^{n+1} n ! \zeta(n+1, s) \quad\left(n \in \mathbb{N} ; s \in \mathbb{C} \backslash \mathbb{Z}_{0}^{-}\right) .
$$

It is also easy to have the following expression ( $c f .[4$, Eq. 1.2(54)]):

$$
\psi^{(m)}(s+n)-\psi^{(m)}(s)=(-1)^{m} m ! H_{n}^{(m+1)}(s-1) \quad\left(m, n \in \mathbb{N}_{0}\right),
$$

which immediately gives $H_{n}^{(s)}$ another expression for $H_{n}^{(s)}$ as follows (cf. [9, Eq. (20)]):

$$
H_{n}^{(m)}=\frac{(-1)^{m-1}}{(m-1) !}\left[\psi^{(m-1)}(n+1)-\psi^{(m-1)}(1)\right] \quad\left(m \in \mathbb{N} ; n \in \mathbb{N}_{0}\right) .
$$

By using finite differences, Spivey [10] presented many summation formulas involving binomial coefficients, the Stirling numbers of the first and second kind and harmonic numbers, two of which are chosen to be recalled here: [10, Identity 14]

$$
\sum_{k=0}^{n}\left(\begin{array}{l}
n \\
k
\end{array}\right) H_{k}=2^{n}\left(H_{n}-\sum_{k=1}^{n} \frac{1}{k 2^{k}}\right) \quad\left(n \in \mathbb{N}_{0}\right),
$$

which was also given by Paule and Schneider [11, Eq. (39)] by deriving it automatically by means of the Sigma package in [12], together with the following identity [10, Iden- 
tity 20]:

$$
\sum_{k=0}^{n}(-1)^{k}\left(\begin{array}{l}
n \\
k
\end{array}\right) H_{k}=-\frac{1}{n} \quad(n \in \mathbb{N})
$$

Paule and Schneider [11] proved five conjectured harmonic number identities similar to those arising in the context of supercongruences for Apéry numbers, one of which is recalled here as follows [11, Eq. (5)]:

$$
\sum_{j=0}^{n}\left(1-5 j H_{j}+5 j H_{n-j}\right)\left(\begin{array}{l}
n \\
j
\end{array}\right)^{5}=(-1)^{n} \sum_{j=0}^{n}\left(\begin{array}{l}
n \\
j
\end{array}\right)^{2}\left(\begin{array}{c}
n+j \\
j
\end{array}\right) .
$$

Greene and Knuth [13, p.10] recorded six commonly used identities that involve both binomial coefficients and harmonic numbers, two of which are recalled here:

$$
\begin{aligned}
& \sum_{j=1}^{n} H_{j}=(n+1) H_{n}-n \quad\left(n \in \mathbb{N}_{0}\right) \\
& \sum_{j=1}^{n}\left(\begin{array}{c}
j \\
m
\end{array}\right) H_{j}=\left(\begin{array}{c}
n+1 \\
m+1
\end{array}\right)\left(H_{n+1}-\frac{1}{m+1}\right) \quad\left(m, n \in \mathbb{N}_{0}\right) .
\end{aligned}
$$

Alzer et al. [14, Eq. (3.62)] proved, by using the principle of mathematical induction, that

$$
\sum_{j=1}^{n} \frac{H_{j}}{j}=\frac{1}{2}\left[\left(H_{n}\right)^{2}+H_{n}^{(2)}\right] \quad(n \in \mathbb{N}) .
$$

By using (1.15) in conjunction with the following elementary identity (see [2]):

$$
H_{j+1}=H_{j}+\frac{1}{j+1}
$$

we obtain

$$
\sum_{j=1}^{n} \frac{H_{j}}{j+1}=\frac{1}{2}\left[\left(H_{n+1}\right)^{2}-H_{n+1}^{(2)}\right] \quad(n \in \mathbb{N}) .
$$

Chu and De Donno [15] made use of the classical hypergeometric summation theorems to derive several striking identities for harmonic numbers other than those discovered recently by Paule and Schneider [11], one of which is recalled below [15, Thereoem 1].

$$
\begin{aligned}
& \sum_{k=0}^{n}\left(\begin{array}{c}
n+\mu n \\
k
\end{array}\right)\left(\begin{array}{c}
n+\lambda n \\
n-k
\end{array}\right) H_{\lambda n+k} \\
& \quad=\left(\begin{array}{c}
2 n+\lambda n+\mu n \\
n
\end{array}\right)\left(H_{\lambda n+n}+H_{\lambda n+\mu n+n}-H_{\lambda n+\mu n+2 n}\right) \quad\left(\lambda, \mu \in \mathbb{N}_{0}\right) .
\end{aligned}
$$

One interesting special case of (1.18) is when we set $\mu=0$. We thus find that

$$
\sum_{k=0}^{n}\left(\begin{array}{l}
n \\
k
\end{array}\right)\left(\begin{array}{c}
n+\lambda n \\
n-k
\end{array}\right) H_{\lambda n+k}=\left(\begin{array}{c}
2 n+\lambda n \\
n
\end{array}\right)\left(2 H_{\lambda n+n}-H_{\lambda n+2 n}\right),
$$


which can be further specialized, with $\lambda=0$, to the following form:

$$
\sum_{k=0}^{n}\left(\begin{array}{l}
n \\
k
\end{array}\right)^{2} H_{k}=\left(\begin{array}{c}
2 n \\
n
\end{array}\right)\left(2 H_{n}-H_{2 n}\right) .
$$

Dattolli and Srivastava [16] proposed several generating functions involving harmonic numbers by making use of an interesting approach based on the umbral calculus. Subsequently, Cvijović [17] showed the truth of the conjectured relations in [16] by using simple analytical arguments.

For a concise and beautiful description of these numbers, we refer also to WolframMathWorld's website [18].

As we have seen in the above brief eclectic review, harmonic and generalized harmonic numbers are involved in a variety of useful identities. Of course, certain interesting properties of harmonic and generalized harmonic numbers have been studied (see, e.g., [19]). Here we aim at presenting further interesting identities about certain interesting finite series associated with binomial coefficients, harmonic numbers and generalized harmonic numbers.

\section{Finite-series involving binomial coefficients, harmonic numbers and generalized harmonic numbers}

As the illustrative identities in Section 1, we consider certain interesting identities about finite-series involving binomial coefficients, harmonic numbers and generalized harmonic numbers. We begin by recalling a known formula ( $c f$. [20, p.362, Entry (55.4.8)]; see also [2, Eq. (2.6)]):

$$
\begin{gathered}
\sum_{j=1}^{n}(-1)^{j} \frac{(a+1)_{j}}{(b+1)_{j}}\left(\begin{array}{l}
n \\
j
\end{array}\right)[\psi(a+1+j)-\psi(a+1)] \\
=\frac{(b-a)_{n}}{(b+1)_{n}}[\psi(b-a)-\psi(b-a+n)] \\
\left(n \in \mathbb{N}_{0} ; a, b \in \mathbb{C} \backslash \mathbb{Z}^{-} ; b-a \in \mathbb{C} \backslash \mathbb{Z}_{0}^{-}\right),
\end{gathered}
$$

where $(\alpha)_{n}$ denotes the Pochhammer symbol defined (for $\alpha \in \mathbb{C}$ ) by

$$
(\alpha)_{n}:= \begin{cases}1 & (n=0) \\ \alpha(\alpha+1) \cdots(\alpha+n-1) & (n \in \mathbb{N})\end{cases}
$$

Differentiating each side of (2.1) with respect to the variables $a$ and $b$, respectively, using (1.8) and considering the following easily derivable identities:

$$
\frac{d}{d \alpha}(\alpha)_{n}=(\alpha)_{n} H_{n}^{(1)}(\alpha-1) \quad\left(n \in \mathbb{N}_{0} ; \alpha \in \mathbb{C} \backslash \mathbb{Z}_{0}^{-}\right)
$$

and

$$
\frac{d}{d \alpha} \frac{1}{(\alpha)_{n}}=-\frac{H_{n}^{(1)}(\alpha-1)}{(\alpha)_{n}} \quad\left(n \in \mathbb{N}_{0} ; \alpha \in \mathbb{C} \backslash \mathbb{Z}_{0}^{-}\right),
$$

we obtain the following formulas in Theorem 1. 
Theorem 1 Each of the following identities holds true:

$$
\begin{aligned}
& \sum_{j=1}^{n}(-1)^{j} \frac{(a+1)_{j}}{(b+1)_{j}}\left(\begin{array}{c}
n \\
j
\end{array}\right)\left[\left\{H_{j}^{(1)}(a)\right\}^{2}-H_{j}^{(2)}(a)\right] \\
& \quad=\frac{(b-a)_{n}}{(b+1)_{n}}\left[\left\{H_{n}^{(1)}(b-a-1)\right\}^{2}-H_{n}^{(2)}(b-a-1)\right] \quad\left(n \in \mathbb{N}_{0} ; a, b \in \mathbb{C} \backslash \mathbb{Z}^{-}\right)
\end{aligned}
$$

and

$$
\begin{aligned}
& \sum_{j=1}^{n}(-1)^{j+1} \frac{(a+1)_{j}}{(b+1)_{j}}\left(\begin{array}{l}
n \\
j
\end{array}\right) H_{j}^{(1)}(a) H_{j}^{(1)}(b) \\
& \quad=\frac{(b-a)_{n}}{(b+1)_{n}}\left[H_{n}^{(2)}(b-a-1)-H_{n}^{(1)}(b-a-1)\left\{H_{n}^{(1)}(b-a-1)-H_{n}^{(1)}(b)\right\}\right] \\
& \quad\left(n \in \mathbb{N}_{0} ; a, b \in \mathbb{C} \backslash \mathbb{Z}^{-}\right) .
\end{aligned}
$$

Setting $a=b-1=0$ in (2.1), (2.5) and (2.6) and using (1.3) and (1.8), we get certain interesting finite-sum identities involving binomial coefficients and harmonic numbers, respectively, asserted by Corollary 1 .

Corollary 1 Each of the following identities holds true:

$$
\begin{aligned}
& \sum_{j=1}^{n} \frac{(-1)^{j+1}}{j+1}\left(\begin{array}{l}
n \\
j
\end{array}\right) H_{j}=\frac{H_{n}}{n+1} \quad\left(n \in \mathbb{N}_{0}\right) \\
& \sum_{j=1}^{n} \frac{(-1)^{j+1}}{j+1}\left(\begin{array}{l}
n \\
j
\end{array}\right)\left[H_{j}^{(2)}-\left(H_{j}\right)^{2}\right]=\frac{1}{n+1}\left[H_{n}^{(2)}-\left(H_{n}\right)^{2}\right] \quad\left(n \in \mathbb{N}_{0}\right)
\end{aligned}
$$

and

$$
\sum_{j=1}^{n} \frac{(-1)^{j+1}}{j+1}\left(\begin{array}{c}
n \\
j
\end{array}\right) H_{j}\left(H_{j+1}-1\right)=\frac{1}{n+1}\left[H_{n}^{(2)}-\frac{n}{n+1} H_{n}\right] \quad\left(n \in \mathbb{N}_{0}\right)
$$

Remark 1 In the course of presenting a closed-form evaluation of some useful series involving the generalized zeta function $\zeta(s, a)$, Choi et al. [21] made use of the identity (2.7) without its proof. Choi and Srivastava [2] proved Eq. (2.7) as a special case of (2.1) here and presented another illustrative proof.

We will try to express a class of the following finite sums involving harmonic numbers and binomial coefficients as given above:

$$
\sum_{j=1}^{n} \frac{(-1)^{j+1}}{(j+1)^{k}}\left(\begin{array}{l}
n \\
j
\end{array}\right) H_{j} \quad\left(n \in \mathbb{N}_{0} ; k \in \mathbb{N}\right)
$$

Here we give the answers for $k=2$ and $k=3$ in (2.10) asserted by the following lemma. 
Lemma 1 Each of the following identities holds true:

$$
\sum_{j=1}^{n} \frac{(-1)^{j+1}}{(j+1)^{2}}\left(\begin{array}{l}
n \\
j
\end{array}\right) H_{j}=\frac{1}{2(n+1)}\left[\left(H_{n+1}\right)^{2}-H_{n+1}^{(2)}\right] \quad\left(n \in \mathbb{N}_{0}\right)
$$

and

$$
\sum_{j=1}^{n} \frac{(-1)^{j+1}}{(j+1)^{3}}\left(\begin{array}{l}
n \\
j
\end{array}\right) H_{j}=\frac{1}{2(n+1)} \sum_{j=1}^{n} \frac{1}{j+1}\left[\left(H_{j+1}\right)^{2}-H_{j+1}^{(2)}\right] \quad\left(n \in \mathbb{N}_{0}\right) .
$$

Proof We will prove only (2.11) by using the same method as in [2, pp.2224-2225]. A similar argument will establish (2.12). We first recall two basic relations for binomial coefficients:

$$
\left(\begin{array}{c}
n+1 \\
j
\end{array}\right)=\left(\begin{array}{c}
n \\
j
\end{array}\right)+\left(\begin{array}{c}
n \\
j-1
\end{array}\right) \text { and }\left(\begin{array}{c}
n \\
j-1
\end{array}\right)=\frac{j}{n+1}\left(\begin{array}{c}
n+1 \\
j
\end{array}\right) .
$$

We let the left-hand side of (2.11) be

$$
f_{n}:=\sum_{j=1}^{n} \frac{(-1)^{j+1}}{(j+1)^{2}}\left(\begin{array}{l}
n \\
j
\end{array}\right) H_{j}
$$

so that, using the first one of (2.13),

$$
\begin{aligned}
f_{n+1}= & \frac{(-1)^{n}}{(n+2)^{2}} H_{n+1} \\
& +\sum_{j=1}^{n} \frac{(-1)^{j+1}}{(j+1)^{2}}\left[\left(\begin{array}{c}
n \\
j
\end{array}\right)+\left(\begin{array}{c}
n \\
j-1
\end{array}\right)\right] H_{j} \\
= & \frac{(-1)^{n}}{(n+2)^{2}} H_{n+1}+f_{n}+\sum_{j=1}^{n} \frac{(-1)^{j+1}}{(j+1)^{2}}\left(\begin{array}{c}
n \\
j-1
\end{array}\right) H_{j} .
\end{aligned}
$$

We now see that, using the second one of (2.13),

$$
\begin{aligned}
& \sum_{j=1}^{n} \frac{(-1)^{j+1}}{(j+1)^{2}}\left(\begin{array}{c}
n \\
j-1
\end{array}\right) H_{j} \\
& \quad=\frac{1}{n+1} \sum_{j=1}^{n}(-1)^{j+1}\left(\begin{array}{c}
n+1 \\
j
\end{array}\right) \frac{j}{(j+1)^{2}} H_{j} \\
& \quad=\frac{1}{n+1}\left[\sum_{j=1}^{n} \frac{(-1)^{j+1}}{j+1}\left(\begin{array}{c}
n+1 \\
j
\end{array}\right) H_{j}-\sum_{j=1}^{n} \frac{(-1)^{j+1}}{(j+1)^{2}}\left(\begin{array}{c}
n+1 \\
j
\end{array}\right) H_{j}\right] .
\end{aligned}
$$

By using the identity in (2.7), we find that

$$
\sum_{j=1}^{n} \frac{(-1)^{j+1}}{j+1}\left(\begin{array}{c}
n+1 \\
j
\end{array}\right) H_{j}=\frac{1-(-1)^{n}}{n+2} H_{n+1} \quad\left(n \in \mathbb{N}_{0}\right) .
$$


We also have

$$
\sum_{j=1}^{n} \frac{(-1)^{j+1}}{(j+1)^{2}}\left(\begin{array}{c}
n+1 \\
j
\end{array}\right) H_{j}=f_{n+1}-\frac{(-1)^{n}}{(n+2)^{2}} H_{n+1} \quad\left(n \in \mathbb{N}_{0}\right) .
$$

Thus, substituting from (2.17) and (2.18) into (2.16), we obtain

$$
\begin{aligned}
& \sum_{j=1}^{n} \frac{(-1)^{j+1}}{(j+1)^{2}}\left(\begin{array}{c}
n \\
j-1
\end{array}\right) H_{j} \\
& \quad=\frac{1}{n+1}\left[\frac{1-(-1)^{n}}{n+2} H_{n+1}-f_{n+1}+\frac{(-1)^{n}}{(n+2)^{2}} H_{n+1}\right] \quad\left(n \in \mathbb{N}_{0}\right) .
\end{aligned}
$$

Finally, it follows from (2.15) and (2.19) that

$$
(n+2) f_{n+1}-(n+1) f_{n}=\frac{H_{n+1}}{n+2} .
$$

Let $a_{n}:=(n+1) f_{n}$ so that we have

$$
a_{n+1}-a_{n}=\frac{H_{n+1}}{n+2} \quad \text { and } \quad a_{1}=\frac{1}{2} H_{1}=\frac{1}{2} .
$$

By telescoping this last sum (2.20), we obtain

$$
a_{n}=(n+1) f_{n}=\sum_{j=1}^{n} \frac{H_{j}}{j+1} .
$$

Applying (1.17) to (2.21), we get the desired identity (2.11).

Applying (2.7) and (2.11) to (2.9) and considering (2.8), we obtain two interesting identities asserted by the following theorem.

Theorem 2 Each of the following identities holds true:

$$
\sum_{j=1}^{n} \frac{(-1)^{j+1}}{j+1}\left(\begin{array}{l}
n \\
j
\end{array}\right)\left(H_{j}\right)^{2}=\frac{1}{2(n+1)}\left[3 H_{n}^{(2)}-\left(H_{n}\right)^{2}\right] \quad\left(n \in \mathbb{N}_{0}\right)
$$

and

$$
\sum_{j=1}^{n} \frac{(-1)^{j+1}}{j+1}\left(\begin{array}{l}
n \\
j
\end{array}\right) H_{j}^{(2)}=\frac{1}{2(n+1)}\left[5 H_{n}^{(2)}-3\left(H_{n}\right)^{2}\right] \quad\left(n \in \mathbb{N}_{0}\right) .
$$

Differentiating (2.5) and (2.6) with respect to $a$ and observing the following identity:

$$
\frac{d}{d \alpha} H_{j}^{(\ell)}(\alpha)=-\ell H_{j}^{(\ell)}(\alpha) \quad(\ell \in \mathbb{N}),
$$

we obtain further interesting identities involving binomial coefficients and generalized harmonic functions asserted by the following theorem. 
Theorem 3 Each of the following identities holds true:

$$
\begin{aligned}
& \sum_{j=1}^{n}(-1)^{j+1} \frac{(a+1)_{j}}{(b+1)_{j}}\left(\begin{array}{l}
n \\
j
\end{array}\right)\left[\left\{H_{j}^{(1)}(a)\right\}^{3}-3 H_{j}^{(1)}(a) H_{j}^{(2)}(a)+2 H_{j}^{(3)}(a)\right] \\
& \quad=\frac{(b-a)_{n}}{(b+1)_{n}}\left[\left\{H_{n}^{(1)}(b-a-1)\right\}^{3}-3 H_{n}^{(1)}(b-a-1) H_{n}^{(2)}(b-a-1)+2 H_{n}^{(3)}(b-a-1)\right] \\
& \left(n \in \mathbb{N}_{0} ; a, b \in \mathbb{C} \backslash \mathbb{Z}^{-}\right)
\end{aligned}
$$

and

$$
\begin{aligned}
& \sum_{j=1}^{n}(-1)^{j+1} \frac{(a+1)_{j}}{(b+1)_{j}}\left(\begin{array}{c}
n \\
j
\end{array}\right) H_{j}^{(1)}(b)\left[\left\{H_{j}^{(1)}(a)\right\}^{2}-H_{j}^{(2)}(a)\right] \\
&= \frac{(b-a)_{n}}{(b+1)_{n}}\left[2 H_{n}^{(3)}(b-a-1)-2 H_{n}^{(1)}(b-a-1) H_{n}^{(2)}(b-a-1)\right. \\
&\left.+\left(\left\{H_{n}^{(1)}(b-a-1)\right\}^{2}-H_{n}^{(2)}(b-a-1)\right)\left\{H_{n}^{(1)}(b-a-1)-H_{n}^{(1)}(b)\right\}\right] \\
&\left(n \in \mathbb{N}_{0} ; a, b \in \mathbb{C} \backslash \mathbb{Z}^{-}\right) .
\end{aligned}
$$

Setting $a=b-1=0$ in (2.25) and (2.26), we find certain interesting identities and using (2.8), respectively, assert the following corollary.

Corollary 2 Each of the following identities holds true:

$$
\begin{aligned}
& \sum_{j=1}^{n} \frac{(-1)^{j+1}}{j+1}\left(\begin{array}{c}
n \\
j
\end{array}\right)\left[\left(H_{j}\right)^{3}-3 H_{j} H_{j}^{(2)}+2 H_{j}^{(3)}\right] \\
& \quad=\frac{1}{n+1}\left[\left(H_{n}\right)^{3}-3 H_{n} H_{n}^{(2)}+2 H_{n}^{(3)}\right] \quad\left(n \in \mathbb{N}_{0}\right)
\end{aligned}
$$

and

$$
\begin{aligned}
& \sum_{j=1}^{n} \frac{(-1)^{j+1}}{j+1}\left(\begin{array}{l}
n \\
j
\end{array}\right)\left(H_{j+1}-1\right)\left[\left(H_{j}\right)^{2}-H_{j}^{(2)}\right] \\
& \quad=\frac{1}{n+1}\left[2 H_{n}^{(3)}-2 H_{n} H_{n}^{(2)}+\frac{n}{n+1}\left\{\left(H_{n}\right)^{2}-H_{n}^{(2)}\right\}\right] \quad\left(n \in \mathbb{N}_{0}\right) .
\end{aligned}
$$

Remark 2 As in getting the results in Theorem 3, it is seen that a variety of interesting identities involving the generalized harmonic numbers can be obtained by applying the differential operator to the parameters of known formulas.

\section{Inverse relations and a question}

By using the known orthogonal relation

$$
\sum_{k=j}^{n}(-1)^{k+j}\left(\begin{array}{l}
n \\
k
\end{array}\right)\left(\begin{array}{l}
k \\
j
\end{array}\right)=\delta_{n j} \quad\left(n \geq j ; n, j \in \mathbb{N}_{0}\right)
$$


with $\delta_{n j}$ the Kronecker delta $\left(\delta_{n n}=1, \delta_{n j}=0\right.$ if $\left.n \neq j\right)$ and a manipulation of double series

$$
\sum_{k=0}^{n} \sum_{j=0}^{k} A_{k, j}=\sum_{j=0}^{n} \sum_{k=j}^{n} A_{k, j}
$$

it is easy to find the following simplest inverse relation (see [22, Chapter 2]):

$$
a_{n}=\sum_{k=0}^{n}(-1)^{k}\left(\begin{array}{l}
n \\
k
\end{array}\right) b_{k} \quad \Leftrightarrow \quad b_{n}=\sum_{k=0}^{n}(-1)^{k}\left(\begin{array}{l}
n \\
k
\end{array}\right) a_{k} .
$$

Applying this inverse relation to the identities in Section 2, we obtain many formulas involving binomial coefficients, harmonic numbers and generalized harmonic numbers asserted by the following corollary.

Corollary 3 Each of the following identities holds true:

$$
\begin{aligned}
& \sum_{j=1}^{n} \frac{(-1)^{j+1}}{j+1}\left(\begin{array}{l}
n \\
j
\end{array}\right)\left[H_{j}^{(2)}-\frac{j}{j+1} H_{j}\right]=\frac{H_{n}\left(H_{n+1}-1\right)}{n+1} \quad\left(n \in \mathbb{N}_{0}\right) ; \\
& \sum_{j=1}^{n} \frac{(-1)^{j+1}}{j+1}\left(\begin{array}{l}
n \\
j
\end{array}\right)\left[\left(H_{j+1}\right)^{2}-H_{j+1}^{(2)}\right]=\frac{2 H_{n}}{(n+1)^{2}} \quad\left(n \in \mathbb{N}_{0}\right) ; \\
& \sum_{j=1}^{n} \frac{(-1)^{j+1}}{j+1}\left(\begin{array}{l}
n \\
j
\end{array}\right)\left[3 H_{j}^{(2)}-\left(H_{j}\right)^{2}\right]=\frac{2\left(H_{n}\right)^{2}}{n+1} \quad\left(n \in \mathbb{N}_{0}\right) ; \\
& \sum_{j=1}^{n} \frac{(-1)^{j+1}}{j+1}\left(\begin{array}{c}
n \\
j
\end{array}\right)\left[5 H_{j}^{(2)}-3\left(H_{j}\right)^{2}\right]=\frac{2 H_{n}^{(2)}}{n+1} \quad\left(n \in \mathbb{N}_{0}\right) ; \\
& \sum_{j=1}^{n} \frac{(-1)^{j+1}}{j+1}\left(\begin{array}{c}
n \\
j
\end{array}\right)\left(H_{j+1}-1\right)\left[2 H_{j}^{(3)}-2 H_{j} H_{j}^{(2)}+\frac{j}{j+1}\left\{\left(H_{j}\right)^{2}-H_{j}^{(2)}\right\}\right] \\
& =\frac{\left(H_{n+1}-1\right)\left[\left(H_{n}\right)^{2}-H_{n}^{(2)}\right]}{n+1} \quad\left(n \in \mathbb{N}_{0}\right) .
\end{aligned}
$$

It is observed that Eqs. (2.7), (2.8) and (2.27) are of the following form:

$$
\sum_{j=1}^{n}(-1)^{j+1}\left(\begin{array}{l}
n \\
j
\end{array}\right) a_{j}=a_{n} \quad(n \in \mathbb{N}) \quad \text { and } \quad a_{0}=0 .
$$

By using the first one of (2.13), we find an identity in the following lemma.

Lemma 2 If Eq. (3.9) holds true, then we obtain the following identity:

$$
\sum_{j=1}^{n}(-1)^{j+1} j\left(\begin{array}{l}
n \\
j
\end{array}\right) a_{j}=n\left(a_{n}-a_{n-1}\right) \quad(n \in \mathbb{N})
$$

Applying Eq. (3.10) to Eqs. (2.7), (2.8) and (2.27), we get some interesting identities asserted by the following corollary. 
Corollary 4 Each of the following identities holds true:

$$
\begin{aligned}
& \sum_{j=1}^{n}(-1)^{j+1} \frac{j}{j+1}\left(\begin{array}{l}
n \\
j
\end{array}\right) H_{j}=\frac{1}{n+1}\left(1-H_{n-1}\right) \quad(n \in \mathbb{N}) ; \\
& \sum_{j=1}^{n}(-1)^{j+1} \frac{j}{j+1}\left(\begin{array}{l}
n \\
j
\end{array}\right)\left[H_{j}^{(2)}-\left(H_{j}\right)^{2}\right] \\
& =\frac{1}{n+1}\left[\left(H_{n}\right)^{2}-H_{n}^{(2)}\right]-\frac{2}{n} H_{n-1} \quad(n \in \mathbb{N}) ; \\
& \sum_{j=1}^{n}(-1)^{j} \frac{j}{j+1}\left(\begin{array}{l}
n \\
j
\end{array}\right)\left[\left(H_{j}\right)^{3}-3 H_{j} H_{j}^{(2)}+2 H_{j}^{(3)}\right] \\
& =\frac{1}{n+1}\left[\left(H_{n}\right)^{3}-3 H_{n} H_{n}^{(2)}+2 H_{n}^{(3)}\right] \quad\left(n \in \mathbb{N}_{0}\right) .
\end{aligned}
$$

Question We conclude this paper by posing a natural question: Under what conditions does Eq. (3.9) hold true?

\section{Competing interests}

The author declares that he has no competing interests.

\section{Acknowledgements}

Dedicated to Professor Hari M. Srivastava.

This work was supported by the Basic Science Research Program through the National Research Foundation of the Republic of Korea funded by the Ministry of Education, Science and Technology (2012-0002957).

Received: 16 November 2012 Accepted: 25 January 2013 Published: 14 February 2013

\section{References}

1. Adamchik, VS, Srivastava, HM: Some series of the zeta and related functions. Analysis 18, 131-144 (1998)

2. Choi, J, Srivastava, HM: Some summation formulas involving harmonic numbers and generalized harmonic numbers. Math. Computer Modelling 54, 2220-2234 (2011)

3. Graham, RL, Knuth, DE, Patashnik, O: Concrete Mathematics. Addison-Wesley, Reading (1989)

4. Srivastava, HM, Choi, J: Series Associated with the Zeta and Related Functions. Kluwer Academic, Dordrecht (2001)

5. Srivastava, HM, Choi, J: Zeta and q-Zeta Functions and Associated Series and Integrals. Elsevier, Amsterdam (2012)

6. Choi, J: Certain summation formulas involving harmonic numbers and generalized harmonic numbers. Appl. Math. Comput. 218, 734-740 (2011). doi:10.1016/j.amc.2011.01.062

7. Rassias, TM, Srivastava, HM: Some classes of infinite series associated with the Riemann zeta and polygamma functions and generalized harmonic numbers. Appl. Math. Comput. 131, 593-605 (2002)

8. Sofo, A, Srivastava, HM: Identities for the harmonic numbers and binomial coefficients. Ramanujan J. 25, 93-113 (2011)

9. Coffey, MW: On some series representations of the Hurwitz zeta function. J. Comput. Appl. Math. 216, 297-305 (2008)

10. Spivey, MZ: Combinatorial sums and finite differences. Discrete Math. 307, 3130-3146 (2007)

11. Paule, P, Schneider, C: Computer proofs of a new family of harmonic number identities. Adv. Appl. Math. 31, 359-378 (2003)

12. Schneider, C: Solving parameterized linear difference equations in $\prod \sum$-fields. Technical Report 02-03, RISC-Linz, J. Kepler University, July 2002. Available at: http://www.risc.uni-linz.ac.at/research/combinat/risc/publications

13. Greene, DH, Knuth, DE: Mathematics for the Analysis of Algorithms, 3rd edn. Birkhäuser, Basel (1990)

14. Alzer, H, Karayannakis, D, Srivastava, HM: Series representations for some mathematical constants. J. Math. Anal. Appl. 320, 145-162 (2006)

15. Chu, W, De Donno, L: Hypergeometric series and harmonic number identities. Adv. Appl. Math. 34, 123-137 (2005)

16. Dattoli, G, Srivastava, HM: A note on harmonic numbers, umbral calculus and generating functions. Appl. Math. Lett. 21, 686-693 (2008)

17. Cvijović, D: The Dattoli-Srivastava conjectures concerning generating functions involving the harmonic numbers. Appl. Math. Comput. 215, 4040-4043 (2010)

18. WolframMathWorld. http://mathworld.wolfram.com/HarmonicNumber.html

19. Conway, JH, Guy, RK: The Book of Numbers. Springer, New York (1996)

20. Hansen, ER: A Table of Series and Products. Prentice-Hall, New Jersey (1975)

21. Choi, J, Cho, YJ, Srivastava, HM: Series involving the zeta function and multiple gamma functions. Appl. Math. Comput. 159, 509-537 (2004)

22. Riordan, J: Combinatorial Identities. Wiley, New York (1968) 
doi:10.1186/1029-242X-2013-49

Cite this article as: Choi: Finite summation formulas involving binomial coefficients, harmonic numbers and generalized harmonic numbers. Journal of Inequalities and Applications 2013 2013:49.

Submit your manuscript to a SpringerOpen ${ }^{\circ}$ journal and benefit from:

- Convenient online submission

- Rigorous peer review

- Immediate publication on acceptance

- Open access: articles freely available online

- High visibility within the field

- Retaining the copyright to your article

Submit your next manuscript at $\gg$ springeropen.com 